\title{
Résumé de la Mise à jour du CCNI concernant I'utilisation recommandée du vaccin contre I'hépatite B
}

\author{
B Henry' ${ }^{1}$ O Baclic ${ }^{2}$ au nom du Comité consultatif national de l'immunisation (CCNI)*
}

\section{Résumé}

Contexte : Des programmes de vaccination des enfants et des adolescents contre l'hépatite B (HB) sont mis en œuvre avec succès dans l'ensemble des provinces et territoires du Canada depuis les années 1990. À la suite de l'introduction de programmes universels de vaccination, l'incidence de I'HB a diminué dans tous les groupes d'âge. Toutefois, demeure inconnue la durée de la protection contre l'infection chronique, telle que mesurée par les cellules mémoire T et $B$.

Objectifs : Passer en revue les données probantes relatives à la protection à long terme contre l'HB chez les adolescents ayant bénéficié de la vaccination systématique au cours de l'enfance, déterminer le niveau de risque d'infection par le virus de l'hépatite B chez les Canadiens diabétiques et déterminer le moment opportun pour revacciner les personnes présentant des affections qui affaiblissent le système immunitaire.

Méthodologie : Le Groupe de travail sur l'hépatite du Comité consultatif national de l'immunisation (CCNI) a passé en revue les principales questions et a effectué un examen et une synthèse des données probantes. En tenant compte des maladies à prévenir, de la population cible et des questions liées à l'innocuité, à l'immunogénicité, à l'efficacité et à l'efficience du vaccin, le groupe de travail a formulé à l'intention du CCNI des recommandations concernant l'utilisation du vaccin. Toutes les données probantes ont été cotées et rapportées dans des tableaux. Le CCNI a approuvé des recommandations précises fondées sur des données probantes et a expliqué leur raison d'être ainsi que les considérations pertinentes dans la mise à jour de la déclaration.

Résultats : Outre les données épidémiologiques, le CCNI a passé en revue les données probantes tirées d'études sur l'efficacité et l'efficience comprenant jusqu'à 30 années de données de suivi, ainsi que les données tirées de 39 publications portant sur la réponse immunitaire après l'administration d'une injection de rappel du vaccin contre l'HB chez des personnes ayant été vaccinées pendant leur enfance. À la lumière de l'examen effectué, le CCNI n'a pas trouvé de données probantes qui appuieraient une modification de sa recommandation actuelle selon laquelle il n'est pas nécessaire de procéder à une vaccination de rappel systématique des personnes qui ont été vaccinées pendant leur enfance, et il n'y a pas non plus de données probantes en appui à un calendrier de vaccination préférentiel ou à une vaccination systématique des personnes diabétiques.

Conclusion : Le CCNI estime maintenant qu'après la vaccination de personnes dont le système immunitaire est affaibli, on peut envisager une surveillance annuelle des niveaux d'anticorps contre le virus de l'HB.

\author{
Affiliations \\ 1 Président du Groupe de travail \\ sur l'hépatite du CCNI, Victoria \\ (Colombie-Britannique) \\ ${ }^{2}$ Centre de l'immunisation et des \\ maladies respiratoires infectieuses \\ (CIMRI), Agence de la santé \\ publique du Canada, Ottawa \\ (Ontario)
}

\author{
*Correspondance : naci-ccni@ \\ phac-aspc.gc.ca
}

Citation proposée : Henry B, Baclic O. Résumé de la Mise à jour du CCNI concernant l'utilisation recommandée du vaccin contre l'hépatite B. Relevé des maladies transmissibles au Canada. 2017;43(5):115-8. https://doi.org/10.14745/ccdr.v43i05a04f

\section{Introduction}

Chez les personnes non vaccinées, le risque d'infection chronique par le virus de l'hépatite $B(\mathrm{HB})$ varie selon l'âge. Jusqu'à $95 \%$ des nourrissons, $50 \%$ des enfants de moins de cinq ans et $10 \%$ des adolescents et adultes développeront une infection chronique (1). Bien que l'on pense que la protection à la suite d'une primovaccination dure longtemps, on ignore quelle est la durée exacte de la protection. Pour une protection à long terme, il faut la présence de cellules mémoire T et B (2-7). À la suite de l'introduction de programmes universels de vaccination dans l'ensemble des provinces et territoires du Canada dans les années 1990, l'incidence de l'HB a diminué dans tous les groupes d'âge (8). On trouve un résumé des recommandations actuelles concernant le vaccin contre l'HB dans le Guide canadien d'immunisation (9).
Le Comité consultatif national de l'immunisation (CCNI) est composé d'experts de l'immunisation provenant des quatre coins du pays et fournit des avis médicaux, scientifiques et en matière de santé publique à l'Agence de la santé publique du Canada (ASPC) sur les vaccins dont la vente est autorisée au Canada. Le Groupe de travail sur l'hépatite du CCNI est composé de membres du CCNI, de membres de liaison et d'autres experts des vaccins qui passent en revue systématiquement - et en faisant la synthèse - les renseignements scientifiques disponibles, ainsi que d'autres renseignements techniques ( $p$. ex., le fardeau de la maladie, les caractéristiques des vaccins, les données d'études non publiées) relatifs à des questions ou à des enjeux précis touchant les vaccins contre I'hépatite $A$ et l'hépatite $B(10)$. 


\section{Méthodologie}

Le Groupe de travail sur l'hépatite a passé en revue les questions principales et a effectué un examen et une synthèse des données probantes relatives à la protection à long terme contre l'HB chez les adolescents qui ont bénéficié d'une vaccination systématique au cours de l'enfance, ainsi que des données probantes relatives au moment opportun pour une revaccination des personnes dont le système immunitaire est affaibli. Le groupe de travail a également passé en revue des données épidémiologiques et de recherche afin de déterminer le niveau de risque d'infection par le virus de I'HB chez les Canadiens diabétiques. En tenant compte du fardeau des maladies à prévenir, de la population cible et des questions liées à l'innocuité, à l'immunogénicité, à l'efficacité et à l'efficience du vaccin, le groupe de travail a formulé à l'intention du CCNI des recommandations concernant I'utilisation du vaccin. Toutes les données probantes ont été cotées et résumées dans des tableaux. Le CCNI a approuvé des recommandations précises fondées sur des données probantes et a expliqué leur raison d'être ainsi que les considérations pertinentes dans la mise à jour de la déclaration.

\section{Résultats}

En 2013, on a rapporté 0,5 cas d'infection aiguë par le virus de l'HB par 100000 habitants et 12 cas d'infection chronique par le virus par 100000 habitants, par l'intermédiaire du Système de surveillance des maladies à déclaration obligatoire du Canada (SSMDO) (10). D'après les données de l'Enquête canadienne sur les mesures de la santé (ECMS), la prévalence de l'infection par le virus de l'HB chez les personnes âgées de 14 à 79 ans est estimée actuellement à $0,4 \%$, et le taux le plus élevé est rapporté dans le cas des populations des non-Blancs (1,8\%) et des personnes nées à l'étranger (1,6\%) (11).

À la lumière d'un examen d'études comprenant jusqu'à 30 années de données, le CCNI n'a pas trouvé de données probantes laissant penser à une efficacité réduite du vaccin à long terme chez les personnes qui ont été vaccinées pendant la prime enfance. Le CCNI a également passé en revue 39 publications contenant des données sur la mémoire immunitaire après l'administration d'une dose de provocation du vaccin contre l'HB, et n'a pas trouvé de données probantes indiquant le besoin d'administrer une dose de rappel de ce vaccin dans le cadre des programmes de vaccination systématique.

À la lumière des données probantes passées en revue, le CCNI a émis quatre recommandations concernant l'utilisation des vaccins contre I'HB au Canada (voir encadré).

\section{Recommandations du CCNI concernant l'utilisation des vaccins contre I'HB au Canada}

Recommandation 1 : Le CCNI ne recommande pas l'administration de doses de rappel systématiques du vaccin contre I'HB chez les personnes immunocompétentes après l'achèvement d'un calendrier d'immunisation contre l'HB recommandé attribué durant la petite enfance. (Recommandation du CCNI selon les données probantes de catégorie B)

Le CCNI conclut qu'il existe des données probantes acceptables pour formuler cette recommandation, d'après le peu de renseignements disponibles au moyen des examens épidémiologiques et de la documentation résumés dans la présente déclaration. Avant la révision des recommandations actuelles, il sera nécessaire de procéder à une évaluation continue et à long terme des données épidémiologiques améliorées concernant l'apparition de maladies aiguës ou l'état de portage de l'antigène HBs dans les populations immunisées (population générale et groupes à risque). Les systèmes nationaux de surveillance améliorée doivent au minimum inclure les renseignements suivants : âge, sexe, comorbidités, vaccination et statut d'immigration.

Recommandation 2 : Le CCNI recommande que les adultes atteints de diabète ne soient pas considérés comme un groupe à risque élevé distinct pour l'immunisation par le vaccin contre l'HB. (Recommandation du CCNI selon les données probantes de catégorie I)

Le CCNI recommande le vaccin contre I'HB pour toutes les personnes ne présentant pas de contre-indications et qui souhaitent réduire leur risque d'HB, y compris les personnes atteintes d'un diabète de type 1 et 2 . Les données américaines semblent indiquer une prévalence plus élevée de l'infection par le VHB antérieure ou actuelle chez les adultes atteints de diabète par rapport aux adultes non diabétiques, mais on ne dispose pas de données épidémiologiques canadiennes similaires. Compte tenu des différences notables entre les systèmes de soins de santé aux États-Unis et au Canada et de l'absence actuelle d'indication de risque plus élevé d'infection pour les personnes atteintes de diabète dans la population canadienne générale, le CCNI ne dispose pas de suffisamment de données probantes pour considérer ces personnes comme un groupe à risque élevé distinct pour l'immunisation par le vaccin à composant anti-hépatite B. Il continuera toutefois à surveiller les données probantes au fil de leur évolution.

Recommandation 3 : Pour les personnes immunodéprimées, on pourrait envisager une surveillance annuelle initiale des niveaux d'anticorps contre le VHB après une immunisation contre I'HB. (Recommandation du CCNI selon les données probantes de catégorie B)

Le moment optimal et la fréquence des tests sérologiques supplémentaires devraient être déterminés en se fondant sur la gravité de l'état d'immunodéficience et selon qu'il existe encore un risque d'HB. Si les titres d'anticorps anti-HBs sont inférieurs à 10 UI/L, les sujets immunodéprimés qui ont répondu au départ au vaccin contre l'HB pourraient avoir besoin de doses de rappel.

Cette recommandation concorde avec des recommandations similaires formulées par l'Advisory Committee on Immunization Practices (ACIP) des États-Unis, I'OMS et le groupe consultatif technique national sur l'immunisation de l'Australie. Le CCNI a déjà recommandé l'évaluation annuelle des niveaux d'anticorps contre le VHB chez les personnes atteintes d'une néphropathie chronique et sous dialyse et qui répondent de façon sous-optimale au vaccin contre l'hépatite B et chez qui les concentrations d'anti-HBs déclinent rapidement. 


\title{
Recommandations du CCNI (suite)
}

\author{
Recommandation $n^{\circ} 4$ : L'immunisation par le vaccin à composant anti-hépatite $B$ devrait être offerte selon des calendriers \\ provinciaux et territoriaux déterminés. (Recommandation du CCNI selon les données probantes de catégorie I)
}

II existe plusieurs calendriers autorisés pour l'administration des vaccins contre l'HB au Canada. Au cours des deux dernières décennies, l'ensemble des PT a mis en œuvre efficacement les programmes de dépistage prénatal de l'HB et d'immunisation des nourrissons à risque

Une réduction marquée de l'incidence de l'hépatite B ayant été observée dans l'ensemble du Canada et compte tenu de l'absence de donnée démontrant l'avantage évident de l'un ou l'autre des calendriers utilisés, le moment optimal de la primovaccination contre I'HB continue d'être subordonné aux considérations liées à des programmes précis et d'épidémiologie des PT. Afin de modifier selon les besoins les programmes d'immunisation contre l'HB existants, on devrait recueillir et analyser de façon continue les renseignements épidémiologiques démontrant l'échec des programmes de dépistage prénatal universel et d'immunisation systématique (c.-à-d. détection d'une infection par le VHB chez les nourrissons et les enfants en attente d'un vaccin).

Un examen complet des données probantes et l'ensemble des recommandations du CCNI sur l'utilisation du vaccin contre l'HB sont publiés dans la mise à jour de la déclaration du CCNI (10) et dans le chapitre sur le vaccin contre I'HB du Guide canadien d'immunisation (9).

\section{Conclusion}

Le CCNI estime maintenant qu'après la vaccination de personnes immunodéprimées, on peut envisager une surveillance annuelle initiale des niveaux d'anticorps contre le virus de l'HB.

\section{Déclarations des auteurs}

La présente déclaration a été préparée par le Groupe de travail sur l'hépatite : $B$. Henry (président du groupe de travail), O. Baclic, J. Brophy, Y. Choudhri, G. Coleman, V. Gilca, S. Ismail, S. Marchant-Short, A. Pham-Huy, M. Salvadori, R. Warrington

\section{Conflit d'intérêts}

Aucun.

\section{Remerciements}

Membres du CCNI : I. Gemmill (président), C. Quach (vice-présidente), N. Dayneka, S. Deeks, B. Henry, S. Marchant-Short, M. Salvadori, N. Sicard, W. Vaudry, D. Vinh, R. Warrington

Représentants de liaison: J. Blake (Société des obstétriciens et gynécologues du Canada), J. Brophy (Association canadienne pour la recherche et l'évaluation en immunisation), A. Cohn (Centers for Disease Control and Prevention, États-Unis), T. Cole (Comité canadien d'immunisation), J. Emili (Collège des médecins de famille du Canada), M. Lavoie (Conseil des médecins hygiénistes en chef), C. Mah (Association canadienne de santé publique), D. Moore (Société canadienne de pédiatrie), A. Pham-Huy (Association pour la microbiologie médicale et I'infectiologie Canada)
Représentants d'office : K. Barnes (Défense nationale et Forces armées canadiennes), G. Charos (Centre de l'immunisation et des maladies respiratoires infectieuses [CIMRI], Agence de la santé publique du Canada [ASPC]), G. Coleman (Direction des produits biologiques et des thérapies génétiques [DPBTG], Santé Canada [SC]), J. Gallivan (Direction des produits de santé commercialisés [DPSC], Santé Canada [SC]), J. Pennock (CIMRI, ASPC), T. Wong (Direction générale de la santé des Premières nations et des Inuits [DGSPNI], SC)

\section{Financement}

Le travail du CCNI est financé par l'Agence de la santé publique du Canada.

\section{Références}

1. Hollinger $F$, Liang TJ. Hepatitis $B$ virus. In: Knipe DM, Howley PM, Griffin DE, Lamb RA, Martin MA, Roizman B, et al, editors. Fields Virology. 4th ed. ed. Philadelphia, PA: Lippincott-Raven Publishers; 2001. p. 2971-3036.

2. Carollo M, Palazzo R, Bianco M, Pandolfi E, Chionne P, Fedele $G$, et al. Hepatitis B specific $T$ cell immunity induced by primary vaccination persists independently of the protective serum antibody level. Vaccine. 2013;31(3):506-13.

3. do Livramento A, Schultz J, Batista KZ, Treitinger A, de Cordova CM, Spada C. Immune memory response induced in vitro by recombinant hepatitis B surface antigen challenge 13-18 years after primary vaccination. J Med Virol. 2014;86(10):1700-4.

4. Rosado MM, Scarsella M, Pandolfi E, Cascioli S, Giorda E, Chionne $\mathrm{P}$, et al. Switched memory B cells maintain specific memory independently of serum antibodies: The hepatitis $B$ example. Eur J Immunol. 2011;41(6):1800-8.

5. Banatvala J, Van Damme P, Oehen S. Lifelong protection against hepatitis $B$ : The role of vaccine immunogenicity in immune memory. Vaccine. 2000;19(7-8):877-85.

6. Bauer T, Jilg W. Hepatitis B surface antigen-specific T and B cell memory in individuals who had lost protective antibodies after hepatitis B vaccination. Vaccine. 2006;24(5):572-7.

7. Simons BC, Spradling PR, Bruden DJ, Zanis C, Case S, Choromanski TL, et al. A longitudinal hepatitis $B$ vaccine cohort demonstrates long-lasting hepatitis B virus (HBV) cellular immunity despite loss of antibody against HBV surface antigen. J Infect Dis. 2016 Apr 7; pii: jiw142. [Diffusion en ligne avant l'impression]. 
8. Gouvernement du Canada [Internet]. Rapport sur l'hépatite B et l'hépatite C au Canada : 2013. Figure 1. Nombre de cas déclarés d'infection aiguë par le VHB au Canada selon le sexe, SSMDO, 2005-2013. Ottawa: ASPC; 2016 [Mise à jour le 28 juil 2016; consulté le 14 mars 2017]. Disponible à l'adresse : http://healthycanadians.gc.ca/publications/ diseases-conditions-maladies-affections/hepatitis-b-c-2013hepatite-b-c/index-fra.php\#f1.

9. Gouvernement du Canada [Internet]. Guide canadien d'immunisation, Partie 4 - Agents d'immunisation active, Vaccin contre I'hépatite B. Ottawa: ASPC; [consulté le 17 mars 2017]. Disponible à l'adresse : https://www.canada. $\mathrm{ca} / \mathrm{fr} /$ sante-publique/services/publications/vie-saine/guidecanadien-immunisation-partie-4-agents-immunisation-active/ page-7-vaccin-contre-hepatite-b.html.
10. Gouvernement du Canada [Internet]. Une déclaration du comité consultatif (DCC) Comité consultatif national de l'immunisation (CCNI). Mise à jour des recommandations concernant l'utilisation du vaccin contre l'hépatite B. Ottawa: ASPC; 2017 [consulté le 17 mars 2017]. Disponible à I'adresse : https://www.canada.ca/fr/sante-publique/services/ publications/vie-saine/mise-a-jour-recommandationsutilisation-vaccin-hepatite-b.html.

11. Statistique Canada [Internet]. Séroprévalence des infections par le virus de I'hépatite $B$ et par le virus de I'hépatite $C$ : résultats de l'Enquête canadienne sur les mesures de la santé, 2007 à 2009 et 2009 à 2011. Ottawa: ASPC; [mise à jour le 27 nov 2015; consulté le 14 mars 2017]. Disponible à l'adresse : http://www.statcan.gc.ca/pub/82-003-x/2013011/ article/11876-fra.htm.

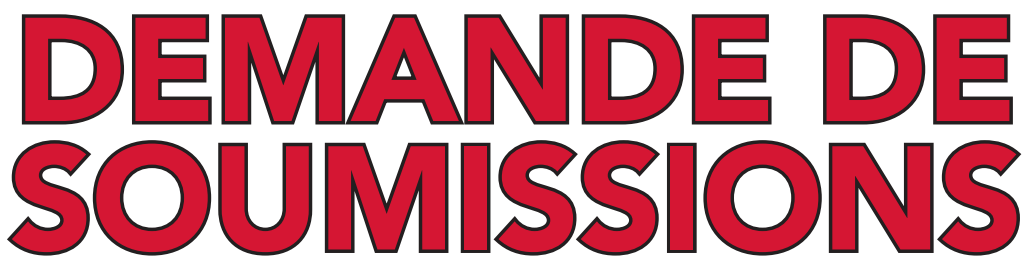

Le saviez-vous? Des articles revus par les pairs sont publiés dans chaque numéro du RMTC en plus de ceux qui portent sur le thème choisi.

\section{SOUMETTEZ DÈS AUJOURD'HUI VOTRE ARTICLE À L'ÉQUIPE DU RMTC!}

- La soumission et la publication d'un article sont GRATUITES (pas de frais de soumission, de publication ou d'évaluation des articles).

- Les articles ne doivent pas nécessairement porter sur le thème particulier d'un numéro

- Pour plus de détails, cliquez sur le lien Soumettre un article dans la page Web du RMTC.

Recherche web: RMTC soumettre un article

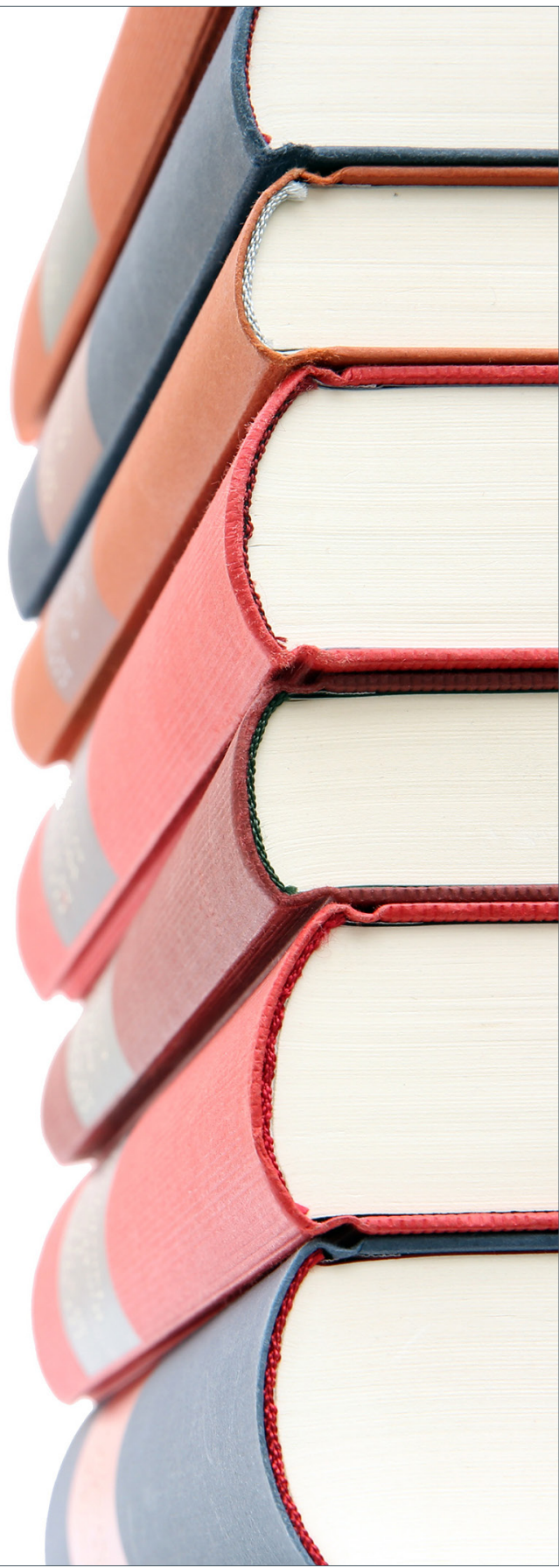

